\title{
Prevalence of Temporomandibular Joint Disorders in Patients with Ankylosing Spondylitis: A Cross-Sectional Study
}

\author{
Rebeca Cecília Souza (D) \\ Emerson Tavares de Sousa (iD ${ }^{2}$ \\ David Sousa (iD ${ }^{3}$ \\ Marcelo Sales (iD) ${ }^{4}$ \\ Rudyard dos Santos Oliveira (iD) 5 \\ Maria Helena Mariano iD ${ }^{6}$ \\ Eliézer Rushansky (iD) ${ }^{7}$ \\ Ana Cláudia Amorim Gomes (iD) ${ }^{8}$ \\ Emanuel Silva (iD) ${ }^{8}$ \\ 'Department of Oral Surgery, Dental \\ School, Pernambuco University, Recife, \\ Pernambuco, Brazil; ${ }^{2}$ Department of \\ Dentistry, Federal University of Rio Grande \\ do Norte, Natal, Rio Grande do Norte, \\ Brazil; ${ }^{3}$ Center of Medical Sciences, Federal \\ University of Paraiba, Joao Pessoa, Paraiba, \\ Brazil; ${ }^{4}$ Department of Clinical and \\ Community Dentistry, Dental School, \\ Federal University of Paraiba, Joao Pessoa, \\ Paraiba, Brazil; ${ }^{5}$ Department of \\ Orthodontics, Sao Leopoldo Mandic School, \\ Campinas, São Paulo, Brazil; ' ${ }^{6}$ epartment of \\ Rheumatology, Oswaldo Cruz University \\ Hospital, Medical School, Pernambuco \\ University, Recife, Pernambuco, Brazil; \\ ${ }^{7}$ Brazilian Society of Rheumatology, \\ Department of Rheumatology, Oswaldo \\ Cruz University Hospital, Medical School, \\ Pernambuco University, Recife, \\ Pernambuco, Brazil; ${ }^{8}$ Department of Oral \\ and Maxillofacial Surgery, Oswaldo Cruz \\ University Hospital, Dental School, \\ Pernambuco University, Recife, \\ Pernambuco, Brazil
}

Correspondence: Emanuel Silva University of Pernambuco, School of Dentistry, Oswaldo Cruz University Hospital, Department of Oral and Maxillofacial Surgery, 310 Arnóbio Marquês Street, Recife, PE, 50I00-130, Brazil

Tel +55 8I-3I84-I468

Email residenciactbmf@upe.br
Background: This study sought to investigate which temporomandibular disorders (TMD) can be expected in patients with ankylosing spondylitis (AS) and to determine the combined impact of these conditions on the psychological status, chronic pain, and functional disability.

Material and Methods: A cross-sectional study composed of 30 patients between 18 and 65 years with ankylosing spondylitis was performed. The research protocol considered the evaluation of outcomes related to the ankylosing spondylitis (HLA-B27 antigen, Bath Ankylosing Spondylitis Disease Activity Index (BASDAI), Bath Ankylosing Spondylitis Functional Index (BASFI) and Health Assessment Questionnaire - Spondylitis (HAQ-S)) and temporomandibular disorders (axis I and II of the Research Diagnostic Criteria for Temporomandibular Disorders - RDC/TMD). Descriptive analyses were applied to express the results.

Results: The sample presented both AS and TMD, most of them (24) were diagnosed with conventional AS (HLA-B27 positive). The BASDAI was scored as 7.70 (2.30) (high activity of AS disease). Functional disability represented by high scores of BASFI [7.00 (2.63)] and HAQ-S [1.79 (0.62)] demonstrates the severe impact of the disease on the daily routine and quality of life. According to RDC/TMD diagnostic criteria, $17(57 \%)$ share the three groups of TMD, and $9(30 \%)$ share two groups of TMD (Group I and III). Over $73 \%$ of the volunteers scored high levels of chronic pain (Grade III and IV) associated with a high depression scale score. The sample scored the somatization scale (with and without pain) as severe.

Conclusion: Patients with ankylosing spondylitis presented a high prevalence of temporomandibular disorder, most of them having the degenerative forms of TMJ disease. AS and TMD cause moderate to severe chronic pain and a negative impact on psychological status and functional capacities.

Keywords: spondyloarthritis, temporomandibular disorders, quality of life, chronic pain

\section{Introduction}

Ankylosing spondylitis (AS) is a chronic and immune-mediated enthesopathy of the axial skeleton that can progress to ossification and ankylosis of fibrocartilaginous joints. ${ }^{1-3}$ Although the estimated prevalence is low $(0.12 \%$ in Latin America and $0.32 \%$ in North America) ${ }^{4}$ the disease is considered a public health problem due to being a potential cause of limitations in daily life, reduced motor activity, emotional impairment, and negative impact on quality of life. ${ }^{5-7}$ 
Some authors argued about the relationship between AS and temporomandibular disorders (TMD). ${ }^{8,9}$ Although not fully understood, destruction of joint capsule or components of the disc, synovitis in TMJ with a breakdown of the articular surfaces, and craniocervical postural changes due to AS are believed to be possible mechanisms underlying the TMJ involvement. Noteworthy, ankylosis is rare in the TMJ probably due to the presence of the intraarticular disc as a physical barrier. ${ }^{10}$

The prevalence of TMD in AS individuals vary across studies, mainly because of the population studied and the method used to assess the TMD. In general, scientific reports that considered the use of imaginological methods and objective and subjective symptoms to diagnose TMD found a prevalence between $30 \%$ and $37 \%{ }^{8,9,11}$ However, these methods did not follow standardized criteria and did not consider a comprehensive evaluation of the TMD. To overcome this deficiency, Research Diagnostic Criteria for Temporomandibular Disorders (RDC/TMD) system was proposed in $1992^{12}$ and updated in 2016 for Diagnostic Criteria for Temporomandibular Disorders (DC/TMD). ${ }^{13}$ In the study of Bilgin et $\mathrm{al}^{5}$ who used the Diagnostic Criteria for Temporomandibular Disorders (DC/TMD) for TMD diagnostic, it was found a TMD prevalence of 59\% in AS individuals. Overall, higher values of the prevalence of TMD in AS individuals were found when the standardized diagnostic criteria were used.

The TMJ is an essential structure for well-performed mastication and other oral functions; thereby, its involvement causes an additional prejudice and vulnerability to individuals who suffer from ankylosing spondylitis. In line with this thought, a better understanding of the clinical manifestations of TMD in AS individuals as well as the combined effect of these conditions on psychosocial variables are relevant to establish a better description of the pattern of co-occurrence and the better approach to overcome severe and incapacitating consequences.

Given this background, this study sought to investigate which temporomandibular disorders can be expected in patients with ankylosing spondylitis and to determine the combined impact of these conditions on the psychological status, chronic pain, and functional disability.

\section{Materials and Methods}

This study was written in consonance with the recommendations of Strengthening the Reporting of Observational Studies in Epidemiology (STROBE) guidelines. ${ }^{14}$

\section{Study Design and Sample Eligibility}

This cross-sectional study was performed in a sample of adults diagnosed with ankylosing spondylitis, who was evaluated between 2017 and 2018. This population was recruited from the Rheumatology Department of the Oswaldo Cruz University Hospital (HUOC), Recife-PE /Brazil. HUOC is a reference hospital for the treatment of ankylosing spondylitis in the Northeast region, especially in the state of Pernambuco (populational density: 9278 million people).

Consecutive sampling recruitment was accomplished by rheumatologists by spontaneous demand. To be eligible, patients must have ankylosing spondylitis diagnosed by HUOC rheumatologists using the modified New York criteria $^{15}$ and aged between 18 and 65 years old. The exclusion criteria were patients: ${ }^{1}$ that did not change the medication in the previous 6 months, ${ }^{2}$ that have any pathology in the TMJ and adjacent tissues identified before ankylosing spondylitis diagnostic, ${ }^{3}$ who is in an acute state of the ankylosing spondylitis, ${ }^{4}$ who undergoing medications that affect bone metabolism, ${ }^{5}$ with neurological or cognitive deficits, ${ }^{6}$ presence of active dental or periodontal pain, and ${ }^{6}$ who had trauma in the head and neck region. Clinical and radiological evaluation for the diagnosis of AS was performed independently by Mariano $\mathrm{MH}$ and Rushansky E. Disagreements between them were resolved by a consensual method.

In the sample, it was evaluated outcomes related to the ankylosing spondylitis, temporomandibular disorders, selfperception of health, and sociodemographic factors. The outcomes centered in ankylosing spondylitis were the serotype of ankylosing spondylitis thought HLA-B27 genetic marker, the disease activity with the Bath Ankylosing Spondylitis Disease Activity Index (BASDAI), and the functional disability using the Bath Ankylosing Spondylitis Functional Index (BASFI) and quality of life using Health Assessment Questionnaire - Spondylitis (HAQ-S). The outcomes related to temporomandibular disorders were the diagnostic of temporomandibular disorders (axis I of the Research Diagnostic Criteria for Temporomandibular Disorders - RDC/TMD), selfreported TMD and bruxism/tightness, psychosocial disability (depression subscales and nonspecific physical symptoms), pain-related intensity and disability, and mandibular functioning limitation (axis II of RDC/TMD). Sociodemographic factors (age, sex, and race) were evaluated using data extracted from axis II of RDC/TMD. 


\section{Sample Size}

Sample size forecast considered the two-sided hypothesis for one sample proportion according to the following formula: ${ }^{16}$

$$
N=\frac{\left(Z_{\frac{\alpha}{2}}+Z_{\beta}\right)^{2} \theta(1-\theta)}{\left(\theta-\theta_{0}\right)^{2}}
$$

Where $\mathrm{Z}$ critical for $\alpha$ was 0.05 (type I error), $\mathrm{Z}$ critical for $\beta$ was 0.20 (type II error), $\theta$ was the expected proportion of temporomandibular disorders in ankylosing spondylitis patients, and $\theta_{0}$ was the proportion of temporomandibular disorders in Brazilian adults. Data from the study of Bilgin et $\mathrm{al}^{5}$ was established for $\theta$, in which TMD was diagnosed in $59.2 \%$ of patients. Otherwise, the study of Progiante et $\mathrm{al}^{17}$ was established for $\theta_{0}$ and found a prevalence of $29.5 \%$ of muscle disorders. Since these authors did not report the overall prevalence (considering disc displacement and other joint disorders), the variable with higher prevalence was chosen (muscle disorders). Therefore, 30 volunteers are required. The calculated number was raised $20 \%$ to avoid sample loss due to non-compliance with the clinical procedures or incomplete answers in the questionnaires.

\section{Data Sources}

\section{HLA-B27 Marker Analysis}

Blood tests for the HLA-B27 marker $^{18}$ were made to differentiate between HLA-B27 positive and HLA-B27 negative ankylosing spondylitis.

\section{Disease Activity of Ankylosing Spondylitis}

Disease activity was measured using the cross-cultural adapted and validated for Brazilian Portuguese speaker's version $^{19}$ of the Bath Ankylosing Spondylitis Disease Activity Index (BASDAI) ${ }^{20}$ This is a self-administered instrument that includes six domains scored from 0 to 10 in a horizontal $10-\mathrm{cm}$ visual analog scale (VAS), in which 10 score means very severe symptoms. Answers considered the last week of the volunteer. The domains assessed were $^{1}$ fatigue,${ }^{2}$ neck, back, and hip pain, ${ }^{3}$ peripheral joint pain and swelling, ${ }^{4}$ localized tenderness, ${ }^{5}$ severity of morning stiffness, and ${ }^{6}$ duration of morning stiffness. The overall score was calculated by the sum of the first four domains with the average of the fifth and sixth. This value was then divided by 5 .

\section{Functional Disability Caused by Ankylosing Spondylitis}

Functional disability was estimated using two indices:

1. The cross-cultural adapted and validated for Brazilian Portuguese speaker's version ${ }^{21}$ of Bath Ankylosing Spondylitis Functional Index (BASFI). ${ }^{22}$ BASFI consists of a self-assessment instrument designed with questions regarding physical function (Q1-8) and the patient's ability to cope with everyday life (Q9-10). Answers considered the last week of the volunteer and were rated in a 10-cm VAS without demarcation. The word "easy" was anchored at the beginning of the line, the word medium at the middle, and the word "impossible" at the end. The overall score was calculated considering the mean of the 10 questions.

2. The short form of the Health Assessment Questionnaire $^{23}$ modified by Daltroy et $\mathrm{al}^{24}$ for use in patients with Spondyloarthropathies (HAQ$\mathrm{S}$ ), and translated, cross-cultural adapted, and validated for use with Brazilian speakers. ${ }^{25}$ This instrument is composed of 20 items across eight categories of daily function as dressing and grooming (Q1-2), arising (Q3-4), eating (Q5-7), walking (Q8-9), hygiene (Q10-12), reach (Q13-14), grip (Q15-17), and common daily activities (Q18-20). Answers considered the last week of the volunteer. The difficulty in performing each activity was scored as without any difficulty (0), with some difficulty, ${ }^{1}$ with much difficulty, and unable to do. ${ }^{3}$ The overall score ranges from 3 (worst) to 0 .

\section{Research Diagnostic Criteria for Temporomandibular Disorders}

Data collection and clinical examination were conducted according to the updated version of RDC/TMD Axis I and Axis II Tests. ${ }^{12}$ This instrument was used considering the translation and adaptation version for Brazilian Portuguese speakers.

Clinical physical examinations (Axis I) were performed to diagnose TMD. This instrument consists of 10 items in which it was performed muscle and joint palpation, testing of mandibular movements, and three subjective questions. Palpations were done with $2 \mathrm{lbs}$ of pressure for extraoral muscles and $1 \mathrm{lb}$ of pressure on the joints and 
intraoral muscles. Axis I was conducted by one calibrated researcher dentist (Souza RV), who was trained by a gold standard examiner in a sample of 10 volunteers. The kappa value was 0.79. Participants were diagnosed in three groups according to the TMD diagnostic algorithm applied to the Axis I of RDC/TMD summary of findings: ${ }^{12}$

- Group I - Muscle Diagnosis: Ia - Myofascial pain; Ib - Myofascial pain with limited opening; Ic - No group I diagnosis.

- Group II - Disc displacements on the (right and left) joint: IIa - Disc displacements with reduction; IIb Disc displacements without reduction, with limited opening; IIc - Disc displacements without reduction, without limited opening; IId - No group II diagnosis.

- Group III - Other joint conditions: IIIa - Arthralgia; IIIb - Osteoarthritis; IIIc - Osteoarthrosis; IIId - No group III diagnosis.

Biobehavioral questionnaire (Axis II) consists of 31 items that comprise the assessment of the following constructs: sociodemographic factors, self-reported TMD, self-perception health, pain-related intensity, and disability (Graded Chronic Pain Scale), Psychosocial disability (depression subscales and nonspecific physical symptoms), and mandibular functioning limitation:

- Sociodemographic factors: Age (Q23), Sex (Q24), and race (Q25).

- Self-reported TMD and bruxism/tightness: Opening of the Jaw (Q14a-b), Click or pop (Q15a), grating or grinding noise $(\mathrm{Q} 15 \mathrm{~b})$, grind or clench the jaw when sleeping (Q15c), grind or clench the jaw during the day (Q15d), stiffness or painful jaw when waking up (Q15e), noise or ringing on the ears (Q15f), and uncomfortable bite feeling $(\mathrm{Q} 15 \mathrm{~g})$. The options available for answers were Yes or No.

- The pain-related intensity and disability (Graded Chronic Pain Scale): First, the Q3 evaluated the presence of chronic pain or not in the last 4 weeks. If no, the volunteer was graded as 0 (no disability). If yes, the Q7-Q9 corresponds to the analysis of Pain intensity; Q10-13 corresponds to the analysis of disability points. Chronic Pain was categorized as Grade I (low disability, low intensity), Grade II (low disability, high intensity), Grade III (high disability, moderately limiting), Grade IV (high disability, severely limiting).
- Psychosocial disability was evaluated using questions related to Depression (Q20: 20 items - b, e, h, i, k, l, m, n, v, y, cc, dd, ee, f, g, q, z, aa, bb, ff), nonspecific physical symptoms with pain-related included (Q20: 12 items - a, c, d, j, o, p, r, s, t, u, $\mathrm{w}, \mathrm{x})$, and nonspecific physical symptoms with pain excluded (Q20: 7 items - c, r, s, t, u, w, x). A minimum of $2 / 3$ answers were required to count the domain for each volunteer. The score for all items answered was: Not at all $=0$; A little bit $=1$; Moderately $=2$; Quite a bit $=3$; Extremely $=4$. The score of each domain was obtained by the sum of the score of each item divided by the number of items answered. Depression index and nonspecific physical symptoms were categorized considering the following cut off points: normal $(<0.535$ : Depression index; $<0.500$ : Nonspecific physical symptoms (pain items included); and <0.428: Nonspecific physical symptoms (pain items excluded)), moderate (0.535-1.105: Depression index; 0.500-1.000: Nonspecific physical symptoms (pain items included); and 0.428-0.857: Nonspecific physical symptoms (pain items excluded)), and severe (>1.105: Depression index; >1.000: Nonspecific physical symptoms (pain items included); and $>0.857$ : Nonspecific physical symptoms (pain items excluded)). ${ }^{26}$

- The mandibular functioning limitation was accessed for item 19 of the questionnaire and provide information regarding subjects' discomfort during some mandibular activities as chewing, drinking, brushing teeth, washing face, exercise, yawning, swallowing, talking, smiling or laughing, and facial expression. Answers were either "yes" or "no". The score of this domain was obtained through the number of positive answers divided by the number of items answered. The number 1 represents the highest score.

The scoring protocol in the Axis II RDC/TMD questionnaire followed the international RDC/TMD consortium. Answers left out of the aforementioned description were excluded.

\section{Data Analysis}

Data were analyzed using the SPSS package for Windows, version 21.0 (SPSS, Inc., Chicago, IL, USA). Descriptive statistics were used to evaluate the quantitative and qualitative variables. For quantitative variables, sample 
distribution was verified using the Shapiro-Wilk test, and data were expressed as mean (standard deviation) or median (Interquartile range) according to the Gaussian distribution. Absolute and percentage frequencies were calculated for categorical variables.

\section{Results}

The study began with 36 volunteers; however, only 30 finished all steps of the research. Table 1 summarizes the sample characteristics, disease activity, and functional disability. The sex ratio male: female was 1.31:1.00 and the

Table I Sample Characteristics, Ankylosing Spondylitis Activity, and Functional Disability

\begin{tabular}{|c|c|}
\hline Age, Yr - Mean (SD) & $49(7.8)$ \\
\hline \multicolumn{2}{|l|}{ Sex $-n^{\circ} .(\%)$} \\
\hline Male & $17(56.7 \%)$ \\
\hline Female & $13(43.3 \%)$ \\
\hline \multicolumn{2}{|l|}{ Race $-\mathbf{n}^{\circ} \cdot(\%)^{\epsilon}$} \\
\hline White & $14(46.7 \%)$ \\
\hline Non-white & $16(53.3 \%)$ \\
\hline \multicolumn{2}{|l|}{ Time Since Diagnosing of AS } \\
\hline Lower than 5 years & $9(30 \%)$ \\
\hline $5-10$ years & $15(50 \%)$ \\
\hline Higher than 10 years & $6(20 \%)$ \\
\hline \multicolumn{2}{|l|}{ Medicine Use - $n^{\circ}$. (\%) } \\
\hline Etanercept & $16(53.3 \%)$ \\
\hline Adalimumab & II (36.7\%) \\
\hline Infliximab & $3(10 \%)$ \\
\hline Positive for HLA-B27 - $n^{\circ}$. (\%) & $24(80 \%)$ \\
\hline \multicolumn{2}{|l|}{ Disease Activity (BASDAI) - Median (IQR) ${ }^{\beta}$} \\
\hline Fatigue & $8.00(3.00)$ \\
\hline Neck, back, and hip pain & $9.00(2.00)$ \\
\hline Peripheral joint pain and swelling & $7.00(4.00)$ \\
\hline Localized tenderness & $8.00(3.00)$ \\
\hline Morning stiffness severity & $8.00(4.00)$ \\
\hline Morning stiffness duration & $5.00(7.00)$ \\
\hline TOTAL SCORE & $7.70(2.30)$ \\
\hline \multicolumn{2}{|l|}{ Functional Disability (BASFI) - Median (IQR) ${ }^{\beta}$} \\
\hline Physical function & $6.25(2.66)$ \\
\hline Ability to cope with everyday life & $8.75(2.50)$ \\
\hline TOTAL SCORE & $7.00(2.63)$ \\
\hline Functional disability (HAQ-S) - Mean (SD) ${ }^{\sigma}$ & $1.79(0.62)$ \\
\hline
\end{tabular}

Notes: ${ }^{\ell}$ Race was self-assessed; ${ }^{\beta}$ Score scale ranged from 10 (worst) to $0 .{ }^{\sigma} S c o r e$ scale ranged from 3 (worst) to 0 . average age of volunteers was 49 (7.8) years. Half of the sample was diagnosed with the disease between 5 and 10 years ago. Except for one volunteer, the sample presented both AS and TMD conditions, most of them ${ }^{24}$ was diagnosed with conventional ankylosing spondylitis (HLAB27 positive). The BASDAI was scored as 7.70 (2.30), which represents the presence of the disease activity. High values of BASFI and HAQ-S demonstrate the severe impact of the disease on the daily routine and quality of life.

Figure 1 demonstrates that only one volunteer did not have TMD according to RDC/TMD diagnostic criteria. Of those individuals affected, 17 (57\%) share the three groups of TMD, and $9(30 \%)$ share two groups of TMD (Group I and III). The most prevalent TMJ subtypes of disorders were myofascial pain with a limited opening (53\%) Group I, disc displacement with reduction (right joint equal to $53 \%$ and left joint equal to $60 \%$ ) - Group II, and arthralgia (right joint equal to $43 \%$ and left joint equal to $50 \%$ ) - Group III (Table 2).

The results in Table 3 indicated that over $73 \%$ of the volunteers scored high levels of chronic pain (Grade III and IV) associated with a high depression scale score (97\% of the sample was graded as moderated or severe depression). The sample scored the somatization scale (with and without pain) as severe.

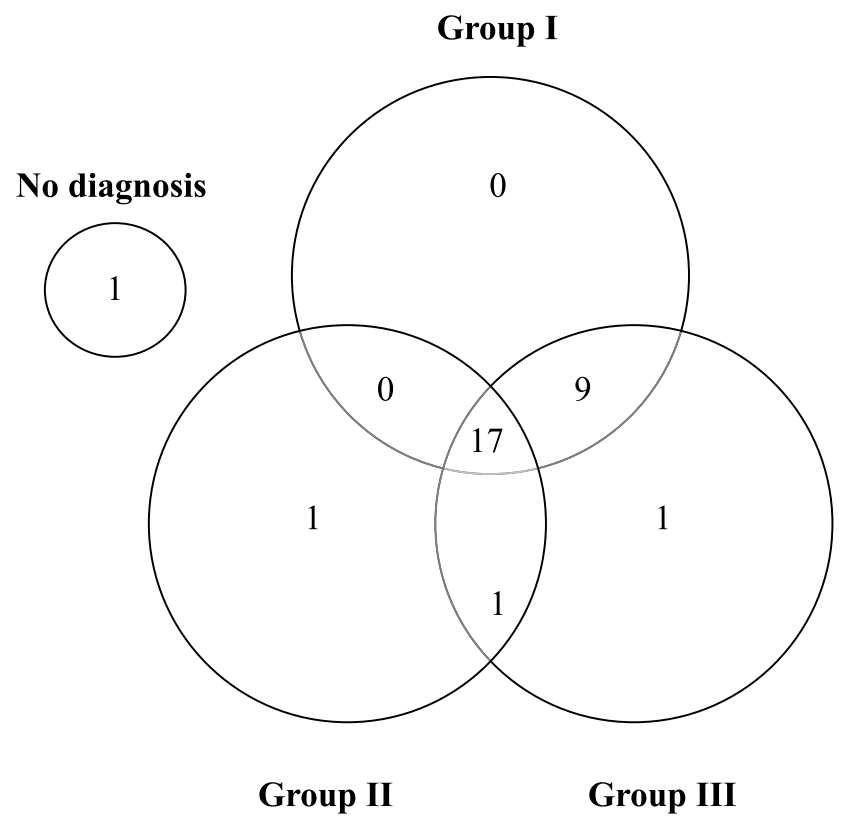

Figure I Diagnostic distributions of the cases relative to TMJ Group I, II, and III. Group I - Muscle Diagnosis, Group II - Disc displacements on the (right and left) joint, and Group III - Other joint conditions (Arthralgia, osteoarthritis, and osteoarthrosis). 
Table 2 Prevalence of TMD Diagnoses According to the Axis I of RDC/TMD

\begin{tabular}{|c|c|c|}
\hline TMJ Subtypes & \multicolumn{2}{|c|}{ Yes n (\%) } \\
\hline \multicolumn{3}{|c|}{ Group I: Muscle Diagnosis } \\
\hline $\begin{array}{l}\text { la: Myofascial pain } \\
\text { lb: Myofascial pain with limited } \\
\text { opening }\end{array}$ & \\
\hline \multicolumn{3}{|c|}{ Group II: Disc Displacements on the Joint } \\
\hline $\begin{array}{l}\text { Ila: Disc displacements with reduction } \\
\text { Illb: Disc displacements without } \\
\text { reduction, with limited opening } \\
\text { Ilc: Disc displacements without } \\
\text { reduction, without limited opening }\end{array}$ & $\begin{array}{c}\text { Right Joint } \\
\text { Yes n (\%) } \\
\text { I6 (53.3) } \\
\text { I (3.3) } \\
0\end{array}$ & $\begin{array}{c}\text { Left Joint } \\
\text { Yes n (\%) } \\
\text { I8 (60) } \\
\text { I (3.3) } \\
0\end{array}$ \\
\hline \multicolumn{3}{|c|}{ Group III: Other Joint Conditions } \\
\hline $\begin{array}{l}\text { Illa: Arthralgia } \\
\text { Illb: Osteoarthritis } \\
\text { Illc: Osteoarthrosis }\end{array}$ & $\begin{array}{l}\text { Right Joint } \\
\text { Yes n (\%) } \\
13(43.3) \\
7(23.3) \\
3(10)\end{array}$ & $\begin{array}{l}\text { Left Joint } \\
\text { Yes n (\%) } \\
15(50) \\
8(26.7) \\
3(10)\end{array}$ \\
\hline
\end{tabular}

Table 3 Prevalence of Pain-Related Intensity and Disability, Psychosocial Disability, and Mandibular Functioning Limitation According to Axis II of RDC/TMD

\begin{tabular}{|c|c|}
\hline \multicolumn{2}{|c|}{ Chronic Pain Grade ${ }^{\mu} n^{\circ} .(\%)$} \\
\hline Grade 0 & $3(10 \%)$ \\
\hline Grade I & $2(6.7 \%)$ \\
\hline Grade II & $6(20 \%)$ \\
\hline Grade III & II (36.7) \\
\hline Grade IV & $8(26.7)$ \\
\hline \multicolumn{2}{|c|}{ Depression $n^{\circ}$. (\%) } \\
\hline Normal & I (3.3\%) \\
\hline Moderate & II (36.7\%) \\
\hline Severe & $18(60 \%)$ \\
\hline \multicolumn{2}{|c|}{ Nonspecific Physical Symptoms $n^{\circ} .(\%)$} \\
\hline \multicolumn{2}{|l|}{ Total Scale } \\
\hline Severe $^{\sigma}$ & $30(100 \%)$ \\
\hline \multicolumn{2}{|c|}{ Pain Excluded $n^{\circ}$. (\%) } \\
\hline Severe $^{\sigma}$ & $30(100 \%)$ \\
\hline Mandibular Limitation - Mean (SD) ${ }^{\beta}$ & $0.36(0.19)$ \\
\hline
\end{tabular}

Notes: ${ }^{\mu}$ Graded Chronic Pain Scale: grade 0, no disability; I, low disability, low intensity; II, low disability, high intensity; III, high disability, moderately limiting; IV, high disability, severely limiting. ${ }^{\circ}$ The categories normal and moderated were not included in the table due to the absence of cases. ${ }^{\beta}$ The worst value for mandibular limitation was I.
When asked regarding their TMD, the jaw locking and limitation in the jaw opening were the less frequent selfreported conditions. The symptoms related to sleep and awake bruxism/tightness as a grind or clench of the jaw when sleep and/or during the day were highly prevalent (Table 4).

Several volunteers considered their general health to be reasonable $(18 / 60 \%)$ or bad $(9 / 30 \%)$. The same trend was observed regarding oral health with $19 / 63 \%$ classified as reasonable and $5 / 17 \%$ as bad. The seek for treatment due to orofacial pain was observed in 3 volunteers $(10 \%)$.

\section{Discussion}

The prevalence of TMD is approximately $5-12 \% ;{ }^{14}$ however, in individuals with AS the prevalence of TMD is seen to be higher. ${ }^{5,8,9}$ This epidemiological phenomenon can be linked with the musculoskeletal nature of both diseases and with the pathophysiology of inflammation in this group of individuals. ${ }^{27}$ In such a scenario, more important than determining the combined prevalence of both diseases is establish into the TMD taxonomy (Group I Muscle Disorders; Group II Disc Displacements; and Group III Arthralgia, Arthritis, Arthrosis) the distribution of TMD subtypes in these individuals and better understand their impact on the psychobiological aspects of life. Noteworthy, we are not able to find any study that provides a comprehensive understanding of the effect of TMD as an AS co-occurrence.

Based on the state-of-the-art in scientific literature and STARD recommendations, ${ }^{28}$ this study used validated methods to analyze AS (New York criteria and BASDAI) and TMD (RDC/TMD) axis I. Besides, the psychosocial impact of these conditions on individuals considered the use of instruments like BASFI and HAQ-S to qualify the functional disability due to AS. RDC/TMD axis II was used to qualify the psychosocial disability, functioning limitation, and chronic pain due to TMD. These instruments were broadly cited in the scientific literature because have a validity of the construct and were translated and validated in several languages, including Brazilian Portuguese.

In the study reported here, it was observed that almost all volunteers with AS have TMD according to RDC/TMD diagnostic criteria and most of them have more than one subtype of TMD $(n=26,87 \%)$. Besides, it was found that a high prevalence of the most incapacitating forms of Group I (Myofascial pain with limited opening: 53\%) and III (Degenerative joint 
Table 4 Prevalence of Self-Reported TMD and Sleep and Awake Bruxism/Tightness According to Axis II of RDC/TMD

\begin{tabular}{|l|c|}
\hline Self-Reported TMD & $\mathbf{n}^{\mathbf{0}}$ Yes (\%) \\
\hline Jaw locking & $10(33.3 \%)$ \\
Limitation in the Jaw opening & $8(26.7 \%)$ \\
Jaw click or pop & $25(83.3 \%)$ \\
Grating or grinding noise & $22(73.3 \%)$ \\
Noise or ringing on the ears & $19(63.3 \%)$ \\
Uncomfortable bite feeling & $27(90 \%)$ \\
Stiffness or painful jaw when waking up & $22(73.3 \%)$ \\
Headaches or migraines & $24(80 \%)$ \\
\hline Bruxism/tightness & \\
\hline Grind or clench the jaw when sleep & $13(43.3 \%)$ \\
Grind or clench the jaw during the day & $19(63.3 \%)$ \\
\hline
\end{tabular}

disease - osteoarthritis and osteoarthrosis: $\approx 35 \%$ ) TMD subtypes. Disk displacement with reduction was the most prevalent phenotype of TMD. When these results were compared with a sample of adults/elderly individuals without AS it was found an overall prevalence of approximately $31 \%$. Disk displacement with reduction was the most prevalent phenotype of TMD with $25.9 \%$. The overall degenerative joint disease (osteoarthritis and osteoarthrosis) was equal to $9.8 \% .{ }^{29}$ Thus, the results of our study demonstrating that AS patients have a trend toward a great severity of TMD disease. Under this context, it must be highlighted that the concomitant occurrence of TMD and AS can increase the morbidity of patients.

Individuals in this research have high disease activity considering the internationally deemed as a BASDAI $\geq$ $4 .{ }^{30}$ Remarkably, patients included in this research use anti-tumor necrosis factor (anti-TNF), which is the currently recommended treatment for patients with persistent disease activity. ${ }^{31}$ A meta-analysis of 14 studies (3186 patients) with low to moderate risk of bias demonstrated that anti-TNF therapy can significantly improve disease activity and physical function in $\mathrm{AS}^{32}$ In this context, caution is needed to interpret our data since the study design did not consider temporality; thus, the influence of any medicine in the improvement in both objective and subjective indicators of disease activity and patient functioning is out of the scope of this research.

The positivity of the HLA-B27 marker $(\mathrm{n}=24,80 \%)$ is in accordance with previous investigations that evidence $80-90 \%$ of the susceptibility to ankylosing spondylitis to the gene for HLA-B27. ${ }^{33}$ Although the mechanism underlying this association is unknown, ${ }^{34}$ HLA-B27 positive patients have more severe disease and systemic manifestation. ${ }^{35}$ Unfortunately, the nature of the research design places some restrictions on the understanding of the HLA-B27 inflammatory marker on the TMJ involvement in patients with AS. Of note, the understanding of the role of the genetic propensity and their respective impact on the clinical manifestation of TMD in AS patients can be elusive.

The functional limitation was assessed using BASFI and HAQ-S. The first one is related to physical function (bending, reaching, changing position, standing, turning, and climbing steps) and the ability to cope with everyday life. The second is related to the impairments in activities of daily living in patients with $\mathrm{AS}^{36}$ Together, these instruments provide a comprehensive understanding of how incapacitating this condition can be. Individuals who suffer from AS have the potential of disability in several important dimensions of human life considering the biopsychosocial health model. Noticeably, the high score for the ability to cope with everyday life (8.75 (SD of 2.50)) can be a signal of an emotionally overwhelmed population.

Chronic pain is an element that attracts attention, mainly because of a high prevalence of the most severe and disabling forms of pain (Grade II to IV equal to 83\%). This result contrasts with the Maringa Study performed in Brazil in a sample of healthy adults in the same country and of similar age. This research pointed out a lower prevalence of chronic pain (36\% of the population regardless of the intensity or limitations due to pain). ${ }^{17}$ To better understand the impact of these outcomes in daily life, the moderate to severe levels of depression $(97 \%$ of the sample) and severe levels of somatization related or not to pain (100\% of the sample) highlight the need for treatment in this group of patients. Here, the call for action to encourage the creation of a comprehensive therapeutic structure that includes the dentist specialized in temporomandibular dysfunction in the rheumatological agenda. Of note, the seek for treatment due to orofacial pain was observed only in three volunteers (10\% of the sample). Besides, in the pain-psychopathology network, the value of cognitive and emotional skills should be enhanced by the presence of mental health professionals.

A high prevalence of jaw-related functional limitation and parafunctional behaviors was found. Moreover, jaw locking and limitation in jaw opening were the less 
frequent self-reported conditions. These results have important implications for dental practice due to the potential complications like a limitation in masticatory function, swallowing, and impairment in social interaction due to prejudice in smiling, talk and sad or painful facial expressions. As a tendency in the literature, restriction of mandibular movements due to ankylosis was not find in any volunteers of our study. Based on a comprehensive image analysis criterion for RDC/TMD images, findings commonly observed in TMJ of AS patients were temporal flattening, abnormal condylar shape, erosions, sclerosis, disk alterations, and osteophytes. ${ }^{37}$ TMJ ankylosis is a rare condition in AS patients probably due to the presence of the articular disc between the bone surface. ${ }^{10}$

Most of the sample report that considers their own oral and general health as reasonable. This outcome must be seemed as relevant mainly in patients who were identified as non-responsive to treatments. Here, the patients' beliefs about their illnesses can restrict adherence with the recommendation of health-care providers and potentially prejudice the compliance with treatment regimens. This trend was observed by Tolu et $\mathrm{al}^{38}$ who found that nearly three out of five AS patients were identified as at risk for nonadherence with the medication regimen. The selfperception of illness can disrupt the cognitive and emotional representations of diseases and point out to a population with a low sense of coherence related to their oral health.

Only three volunteers seek treatment due to orofacial pain. The key element above this outcome should be further investigated to improve treatment strategies targeted to this population. We can speculate three main reasons for this finding. Firstly, the co-occurrence of pain in other articulations possibly hides the importance of TMJ involvement. Secondly, the cultural low importance related to oral health when together with a medical problem. Thirdly, the lack of proper information regarding the own disease and the potential co-occurrence of TMD (as a separated disease entity).

This study has some strengths and limitations. Studies that consider psychosocial aspects of both AS and TMD are scarce in the scientific literature; thus, this study made a significant worldwide contribution to this research area and provide further background to the Brazilian Registry of Spondyloarthritides. Additionally, the use of validated instruments improves the quality of data, comparability with other realities, and provides a source for future research.
Cautionary notes include the lack of a control group with characteristics of individuals without rheumatic diseases, the cross-sectional design limits aspects related to the causality of TMD as an AS co-occurrence. Moreover, as this study only considered patients that used anti-TNF medicines, there is an overall heavy disease burden ${ }^{31}$ which may be a source of serious selection bias as the recruitment considered a hospital-based convenience sample. In this context, caution is needed to interpret our data since the study design did not consider temporality; thus, the influence of any medicine in the improvement in both objective and subjective indicators of disease activity and patient functioning is out of the scope of this research.

To overcome these limitations in a more complete clinical and research perspective, longitudinal and prospective cohort or case-control studies with a larger and randomized sample can be elusive to establish the potential psychosocial markers and better predict the natural course of TMD in individuals who suffer from AS.

\section{Conclusions}

Patients with ankylosing spondylitis presented a high prevalence of temporomandibular disorder, most of them having the degenerative forms of TMJ disease, bilaterally. AS and TMD cause moderate to severe chronic pain and a negative impact on psychological status and functional capacities.

\section{Ethics and Consent}

This study was approved by the Research Ethics Committee of the State University of Pernambuco - UPE (CAAE 15966819.1.0000.5207), which followed the Declaration of Helsinki and the regulatory guidelines and norms obey 466/ 12 resolution for research ethics in Brazil. All individuals who agreed to participate in this study signed an informed consent form, authorizing their participation.

\section{Acknowledgments}

We especially thank all volunteers for their valuable contribution to our research.

\section{Author Contributions}

All authors contributed to data analysis, drafting or revising the article, gave final approval of the version to be published, agreed to the submitted journal, and agree to be accountable for all aspects of the work. 


\section{Funding}

The first-listed author was granted a Ph.D. Scholarship from Coordination from the Improvement of Higher Education Personnel (CAPES, Ministry of Education, Brazil).

\section{Disclosure}

All authors declare that they have no conflicts of interest for this work.

\section{References}

1. Dougados M, Baeten D. Spondyloarthritis. Lancet. 2011;377 (9783):2127-2137. doi:10.1016/S0140-6736(11)60071-8

2. Braun J, Sieper J. Ankylosing spondylitis. Lancet. 2007;369:1379-1390.

3. Zhu W, He X, Cheng K, et al. Ankylosing spondylitis: etiology, pathogenesis, and treatments. Bone Res. 2019;7:22.

4. Dean LE, Jones GT, MacDonald AG, Downham C, Sturrock RD, Macfarlane GJ. Global prevalence of ankylosing spondylitis. Rheumatology (Oxford). 2014;53:650-657.

5. Bilgin E, Bilgin E, Özdemir O, Kalyoncu U. Temporomandibular disorders in ankylosing spondylitis: a cross-sectional, monocentric study. Rheumatol Int. 2020;40:933-940.

6. Alkan H, Yildiz N, Ardiç F. The correlations between disease specific quality of life, short form-36 and clinical variables in patients with ankylosing spondylitis. Arch Rheumatol. 2020;35:468-476.

7. Mogard E, Olofsson T, Bergman S, et al. Chronic pain and assessment of pain sensitivity in patients with axial spondyloarthritis: results from the SPARTAKUS cohort. $J$ Rheumatol. 2020; jrheum.200872. doi:10.3899/jrheum.200872

8. Helenius LM, Hallikainen D, Helenius I, et al. Clinical and radiographic findings of the temporomandibular joint in patients with various rheumatic diseases. A case-control study. Oral Surg Oral Med Oral Pathol Oral Radiol Endod 2005;99:455-463.

9. Yildizer Keris E, Yaman SD, Demirag MD, Haznedaroglu S. Temporomandibular joint findings in patients with rheumatoid arthritis, ankylosing spondylitis, and primary Sjogren's syndrome. J Investig Clin Dent. 2017;8:12255.

10. Shadamarshan Rengasayee A, Roy Chowdhury SK, Sharma R, Padma Priya S. Novel hypotheses related to Temporomandibular joint derived from Ankylosing spondylitis. Med Hypotheses. 2020;144:110225.

11. Ramos-Remus C, Major P, Gomez-Vargas $\mathrm{A}$, et al. Temporomandibular joint osseous morphology in a consecutive sample of ankylosing spondylitis patients. Ann Rheum Dis. 1997;56:103-107.

12. Dworkin SF, LeResche L. Research diagnostic criteria for temporomandibular disorders: review, criteria, examinations and specifications, critique. J Craniomand Disord. 1992;6:301-355.

13. Schiffman E, Ohrbach R; International RDC/TMD Consortium Network, International association for Dental Research; Orofacial Pain Special Interest Group, International Association for the Study of Pain, et al. Diagnostic Criteria for Temporomandibular Disorders (DC/TMD) for Clinical and Research Applications: Recommendations of the International RDC/TMD Consortium Network* and Orofacial Pain Special Interest Group †. J Oral Facial Pain Headache. 2014;28:6-27.

14. von Elm E, Altman DG, Egger M, Pocock SJ, Gøtzsche PC Vandenbroucke JP; STROBE Initiative. The Strengthening the Reporting of Observational Studies in Epidemiology (STROBE) Statement: guidelines for reporting observational studies. Int J Surg. 2014;12:1495-1499.
15. van der Linden S, Valkenburg HA, Cats A. Evaluation of diagnostic criteria for ankylosing spondylitis: a proposal for modification of the New York criteria. Arthritis Rheum. 1984;27:361-368.

16. Chow S, Wang. Sample Size Calculations in Clinical Research. NY: Taylor \& Francis; 2003:82-83.

17. Progiante PS, Pattussi MP, Lawrence HP, Goya S, Grossi PK, Grossi ML. Prevalence of temporomandibular disorders in an adult Brazilian community population using the research diagnostic criteria (Axes I and II) for temporomandibular disorders (The Maringá Study). Int J Prosthodont. 2015;28:600-609.

18. Dominguez O, Coto E, Martinez-Naves E, Choo SY, Lopez-Larrea C. Molecular typing of HLA-B27 alleles. Immunogenetics. 1992;36:277-282.

19. Pimentel-Santos FM, Pinto T, Santos H, et al. Portuguese version of the bath indexes for ankylosing spondylitis patients: a cross-cultural adaptation and validation. Clin Rheumatol. 2012;31:341-346.

20. Garrett S, Jenkinson T, Kennedy LG, Whitelock H, Gaisford P, Calin A. A new approach to defining disease status in ankylosing spondylitis: the Bath Ankylosing Spondylitis disease activity index. J Rheumatol. 1994;21:2286-2291.

21. Cusmanich KG, Kowalski SC, Gallinaro AL, GoldensteinSchainberg C, Souza LA, Gonçalves CR. Cross-cultural adaptation and validation of the Brazilian-Portuguese version of the Bath Ankylosing Spondylitis Functional Index (BASFI). Rev Bras Reumatol. 2012;52:733-741.

22. Calin A, Garrett S, Whitelock H, et al. A new approach to defining functional ability in ankylosing spondylitis: the development of the Bath Ankylosing Spondylitis functional index. $J$ Rheumatol. 1994;21:2281-2285.

23. Fries JF, Spits P, Kraines RG, Holman HR. Measurement of patient outcome in arthritis. Arthritis Rheum. 1980;23:137-145.

24. Daltroy LH, Larson MG, Roberts NW, Liang MH. A modification of the Health Assessment Questionnaire for the spondyloarthropathies. $J$ Rheumatol. 1990;17:946-950.

25. Shinjo SK, Gonçalves R, Kowalski S, Gonçalves CR. BrazilianPortuguese version of the Health Assessment Questionnaire for Spondyloarthropathies (HAQ-S) in patients with ankylosing spondylitis: a translation, cross-cultural adaptation, and validation. Clin Rheumatol. 2007;26:1254-1258.

26. Kim YK, Kim SG, Im JH, Yun PY. Clinical survey of the patients with temporomandibular joint disorders, using Research Diagnostic Criteria (Axis II) for TMD: preliminary study. J Craniomaxillofac Surg. 2012;40:366-372.

27. Taurog JD, Chhabra A, Colbert RA. Ankylosing spondylitis and axial spondyloarthritis. $N$ Engl J Med. 2016;375:1303.

28. Bossuyt PM, Reitsma JB; Standards for Reporting of Diagnostic Accuracy, et al.. Towards complete and accurate reporting of studies of diagnostic accuracy: the STARD initiative. Clin Radiol. 2003;58:575-580.

29. Valesan LF, Da-cas CD, Réus JC, et al. Prevalence of temporomandibular joint disorders: a systematic review and meta-analysis. Clin Oral Investig. 2021;25:441-453.

30. Cohen JD, Cunin P, Farrenq V, et al. Estimation of the bath ankylosing spondylitis disease activity index cutoff for perceived symptom relief in patients with spondyloarthropathies. $J$ Rheumatol. 2006;33:79-81.

31. van der Heijde D, Sieper J; Assessment of SpondyloArthritis international Society, et al. 2010 Update of the international ASAS recommendations for the use of anti-TNF agents in patients with axial spondyloarthritis. Ann Rheum Dis. 2011;70:905-908.

32. Zong HX, Xu SQ, Tong H, Wang XR, Pan MJ, Teng YZ. Effect of anti-tumor necrosis factor $\alpha$ treatment on radiographic progression in patient with ankylosing spondylitis: a systematic review and metaanalysis. Mod Rheumatol. 2019;29:503-509.

33. Thomas GP, Brown MA. Genetics and genomics in ankylosing spondylitis. Immunol Rev. 2010;233:162-180. 
34. Taurog JD. The role of HLA-B27 in spondyloarthritis. J Rheumatol. 2010;37:2606-2616.

35. Usha SGK. Role of HLA B27 in diagnosis of seronegative spondyloarthropathies. Indian J Pathol Microbiol. 2007;50:908-913.

36. Zochling J. Measures of symptoms and disease status in ankylosing spondylitis: ankylosing Spondylitis Disease Activity Score (ASDAS), Ankylosing Spondylitis Quality of Life Scale (ASQoL), Bath Ankylosing Spondylitis Disease Activity Index (BASDAI), Bath Ankylosing Spondylitis Functional Index (BASFI), Bath Ankylosing Spondylitis Global Score (BAS-G), Bath Ankylosing Spondylitis Metrology Index (BASMI), Dougados Functional Index (DFI), and Health Assessment Questionnaire for the Spondylarthropathies (HAQ-S). Arthritis Care Res (Hoboken). 2011;63(Suppl 11):S47-58.
37. Ahmad M, Hollender L, Anderson Q, et al. Research diagnostic criteria for temporomandibular disorders (RDC/TMD): development of image analysis criteria and examiner reliability for image analysis. Oral Surg Oral Med Oral Pathol Oral Radiol Endod. 2009;107:844-860.

38. Tolu S, Rezvani A, Karacan İ, et al. Self-reported medication adherence in patients with ankylosing spondylitis: the role of illness perception and medication beliefs. Arch Rheumatol. 2020;35:495-505.

\section{Publish your work in this journal}

Clinical, Cosmetic and Investigational Dentistry is an international, peer-reviewed, open access, online journal focusing on the latest clinical and experimental research in dentistry with specific emphasis on cosmetic interventions. Innovative developments in dental materials, techniques and devices that improve outcomes and patient satisfaction and preference will be highlighted. The manuscript management system is completely online and includes a very quick and fair peer-review system, which is all easy to use. Visit http://www.dovepress.com/testimonials.php to read real quotes from published authors. 